\title{
A US Time and Motion Pilot Study of Nebulized COPD Therapy in an Inpatient and a Long-Term Care Setting
}

Erwin De Cock · Grace Leung · Grant Maclaine · Hemal Shah •

Brooks Kuhn · Bryan Nichols

Received: November 9, 2021 / Accepted: February 18, 2022 / Published online: March 4, 2022

(c) The Author(s) 2022

\section{ABSTRACT}

Introduction: There is a lack of quantitative data on healthcare professionals' (HCPs) time dedicated to nebulized chronic obstructive pulmonary disease (COPD) therapy in inpatient and long-term care (LTC) settings. Using time and motion methodology, we quantified HCP time and opportunity costs (time and materials) associated with nebulized COPD therapy in inpatient and LTC settings and estimated efficiencies of changing to once-daily therapy.

Methods: A case report form was built to reflect local nebulization workflow. Primary outcomes were mean active HCP time per predefined task

E. De Cock $(\bowtie)$

Syneos Health, Carrer Pau Claris, 196, 5 08037

Barcelona, Spain

e-mail: erwin.decock@syneoshealth.com

G. Leung

Theravance Biopharma US, Inc., South San

Francisco, CA, USA

G. Maclaine

Theravance Biopharma Ireland Limited, Dublin, Ireland

H. Shah

Value Matters, LLC, Ridgefield, CT, USA

B. Kuhn

UC Davis School of Medicine, Sacramento, CA, USA

B. Nichols

Intrafil Rx LLC, Salt Lake City, UT, USA and mean total active HCP time associated with short-acting beta agonist (SABA) and SABA/ short-acting muscarinic antagonist (SAMA) combination nebulization processes.

Results: Twenty observations occurred within each setting. Inpatient observations included three SABA and 17 SABA/SAMA (from 18 different patients), and LTC observations included five SABA and 15 SABA/SAMA (from eight different patients). Mean total process time was $16.12 \mathrm{~min}$ in the inpatient setting (95\% CI 14.48-17.76) and $21.0 \mathrm{~min}$ in the LTC setting $(95 \% \mathrm{CI}$ 18.8-23.2), with the actual nebulization comprising over $50 \%$ of process time for both. In LTC, CIs suggest a difference by cognitive impairment status: mean $24.1 \mathrm{~min}$ (95\% CI 21.3-26.9) if cognitively impaired versus $19.0 \mathrm{~min}(95 \% \mathrm{CI}$ 16.1-21.8) if not. In the inpatient setting, the estimated process time/admission was $7.8 \mathrm{~h}$; a once-daily nebulized drug would require only $2.3 \mathrm{~h}$. In LTC, the estimated process time was $32.1 \mathrm{~h} /$ month; a once-daily nebulized drug would require only $13.7 \mathrm{~h} / \mathrm{month}$. Estimated nebulization cost was $\$ 243 /$ admission for current versus $\$ 72$ for once-daily dosing in inpatient, and $\$ 1177 /$ month versus $\$ 504$ in LTC.

Conclusions: The nebulization process for COPD patients in both inpatient and LTC settings consumes considerable HCP time. A switch from SABA or SABA/SAMA to a drug with a once-daily nebulization frequency could generate substantial time savings depending on the setting and site characteristics. 
Keywords: Albuterol sulfate; Cost; Ipratropium bromide; Process workflow; Nebulization

\section{Key Summary Points}

Why carry out this study?

Growing shortages of nurses and respiratory therapists are expected in the US, resulting in increasing demands on the existing workforce.

There is a lack of quantitative data on healthcare professionals' (HCPs') time dedicated to nebulized chronic obstructive pulmonary disease (COPD) therapy in inpatient and long-term care (LTC) settings.

In this pilot study, using time and motion methodology, we quantified HCP time and opportunity costs (time and materials) associated with nebulized COPD therapy in inpatient and LTC settings and estimated efficiencies of changing to once-daily therapy.

\section{What was learned from the study?}

Findings from this pilot study validate the use of the time and motion methodology and suggest that switching from a shortacting beta agonist (SABA) or SABA/shortacting muscarinic antagonist (SAMA) to a once-daily nebulized drug could generate a sizable reduction in process time per admission or month of stay, depending on the setting and site characteristics.

A switch from SABA or SABA/SAMA to a drug with a once-daily nebulization frequency could generate substantial time savings, depending on the setting of care and patient characteristics.

Expanding this pilot study by adding additional sites and increasing the overall sample size would increase the precision of the results and make them more generalizable to inpatient and LTC settings across the US.

\section{INTRODUCTION}

As of 2018, an estimated 16.4 million adults had a diagnosis of chronic obstructive pulmonary disease (COPD) in the United States (US), which represents $6.6 \%$ of adults, with millions more who are undiagnosed [1]. Although most COPD therapies are delivered by the inhaled route via handheld devices, certain patients (e.g., those with suboptimal peak inspiratory flow, cognitive impairment, or dexterity limitations) may benefit from nebulized therapy [2]. Commonly used nebulized COPD therapies include the short-acting beta agonist (SABA) albuterol sulfate and the fixed-dose SABA/short-acting muscarinic antagonist (SAMA) combination albuterol sulfate/ipratropium bromide. Both are typically administered every $4-6 \mathrm{~h}$.

Growing shortages of nurses and respiratory therapists are expected in the US, resulting in increasing demands on the existing workforce [3]. There is a lack of quantitative data on healthcare professionals' (HCPs) time dedicated to nebulized COPD therapy in US inpatient and long-term care (LTC) settings. Previous workflow mapping of nebulized COPD therapy in those settings confirmed that (1) workflow was highly standardized and consistent across both settings and bronchodilators and (2) time and motion (T\&M) methodology is suitable to accurately quantify HCP time for nebulization process [4].

Our objectives in this pilot study were to quantify HCP time and costs (of HCP time and materials) associated with nebulized albuterol sulfate (SABA) and albuterol sulfate/ipratropium bromide (SABA/SAMA) in inpatient and LTC settings in the US and to estimate potential time and cost efficiencies that could result from newer therapies with less frequent dosing regimens.

\section{METHODS}

This was a prospective observational T\&M study. T\&M methodology consists of (1) breaking down a process into its main tasks and (2) repeated measurements of the duration of those tasks. First, one HCP from each site completed a 
survey to describe center characteristics, COPD treatment, and nebulization workflow focused on SABA and SABA/SAMA administration. Subsequently, a case report form was built to reflect local nebulization workflow. Primary outcome measures were mean active HCP time per predefined task and mean total active HCP time associated with SABA and SABA/SAMA nebulization processes. All patients with a diagnosis of acute COPD exacerbation (inpatient setting) or chronic COPD (LTC setting) were candidates for observation. Institutional review board approval for 20 observations was obtained at each site, and informed consent was not required because limited patient-level data (sex, COPD severity, cognitive status) were collected and the focus of data collected was provider time. All data were anonymized. Random nebulization processes were observed, which could involve the same patients.

Descriptive statistics for this study were calculated as mean, standard deviation, 5\% and 95\% quantiles (predefined tasks), and 95\% confidence interval (CI) (total process time) for continuous variables. Costs (HCP time and materials) were calculated using publicly available hourly salary data for HCPs for 2020, reported by the US Bureau of Labor Statistics $[5,6]$ and local purchase prices for materials (tubing, mask, mouthpiece). Time and costs were calculated for three scenarios: SABA alone, SABA/SAMA alone, SABA/SAMA + SABA PRN (as needed). Weighted results (applying the distribution across scenarios) are presented. Analyses were performed using $\mathrm{SAS}^{\odot}$ version 9.1.3 (SAS Institute, Cary, NC, USA) and Microsoft ${ }^{\circledR}$ Office Excel ${ }^{\circledR} 2007$.

Institutional review board approval for 20 observations was obtained at each site; informed consent was not required because limited patient-level data (sex, COPD severity, cognitive status) were collected, and the focus of data collected was provider time. All data were anonymized. The study was performed in accordance with the Helsinki Declaration of 1964 and its later amendments.

\section{RESULTS}

\section{Sample Characteristics}

A total of 20 observations occurred within each setting. Inpatient observations included three SABA and 17 SABA/SAMA (from 18 different patients), and LTC observations included five SABA and 15 SABA/SAMA (from eight different patients). Eight observations in the LTC group were from cognitively impaired patients. In the LTC setting, $38 \%$ of the patients who were observed were short-term residents (e.g., recovery after surgery), and $62 \%$ were long-term (staying indefinitely) residents. At the inpatient site, clinician opinion using Global Initiative for Chronic Obstructive Lung Disease (GOLD) 2019 guidelines characterized COPD severity of observed patients as $11 \%$ mild, $17 \%$ moderate, $44 \%$ severe, and $28 \%$ very severe (and estimated the typical distribution of patients admitted to the unit with COPD exacerbation as $0 \%$ mild, $20 \%$ moderate, $50 \%$ severe, and $30 \%$ very severe). At the LTC site, clinician opinion characterized the COPD severity of observed patients as 50\% moderate and 50\% severe (and estimated the current distribution of the site's COPD population by severity as $40 \%$ mild, 30\% moderate, $10 \%$ severe, and $20 \%$ very severe).

\section{Time per Patient Nebulization Process}

For the inpatient site, mean total process time across the nine steps of the nebulization process was $16.12 \min$ (95\% CI 14.48-17.76), of which $50 \%$ was actual nebulization time (Fig. 1). All tasks were performed by a respiratory therapist (RT).

For the LTC site, mean total process time was $21.01 \mathrm{~min}$ (95\% CI 18.80-23.22), of which $67 \%$ was actual nebulization time. CIs suggest a difference by cognitive impairment status in total nebulization process time: mean $24.06 \mathrm{~min}$ (95\% CI 21.29-26.84) if cognitively impaired versus $18.98 \mathrm{~min}$ (95\% CI 16.12-21.83) if not cognitively impaired. Process time weighted by cognitive impairment status $(71 \%$ of COPD patients at any given time were reportedly impaired and 29\% not, on the basis of clinician 


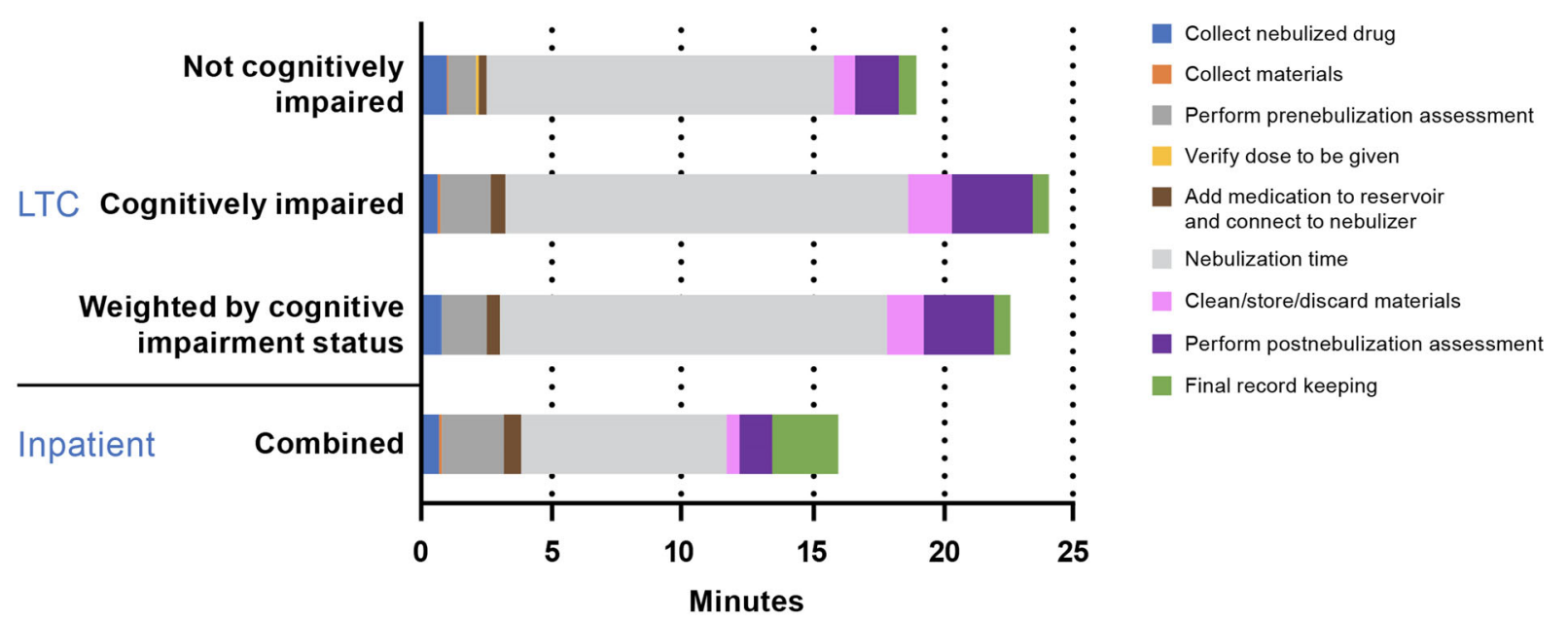

Fig. 1 Mean time per nebulization process (active HCP time only). HCP healthcare professional, LTC long-term care

opinion) would be $22.59 \mathrm{~min}$ (Fig. 1). All tasks were performed by a registered nurse (RN).

\section{Nebulization Process Time per Admission (Inpatient) and per Patient per Month (LTC)}

For both the inpatient and LTC sites, per interview reports, daily nebulization frequency of SABA/SAMA varied by COPD severity, and SABA PRN was expected to be given once a day. For the inpatient site, weighted average frequency per day was 4.8 for SABA/SAMA (calculated as the reported distribution by COPD severity at admission multiplied by the expected nebulization frequency: 1.5 for mild, 2.5 for moderate, 5.0 for severe, and 6.0 for very severe COPD). Mean nebulization process time per admission (reported as 5.4 days on average), weighted by treatment scenario (40\% receiving SABA/SAMA alone, $60 \%$ SABA/SAMA + SABA PRN), was 7.8 h (Fig. 2a). A once-daily nebulized drug (40\% alone and 60\% with once-daily SABA PRN) would require $2.3 \mathrm{~h}$ of nebulization process time per admission, a 70\% reduction (Fig. 2a).

For the LTC site, weighted average frequency per day was 2.6 for either SABA or SABA/SAMA (calculated as the reported distribution by COPD severity multiplied by the expected nebulization frequency: 1.0 for mild, 2.0 for moderate, 4.0 for severe, and 6.0 for very severe
COPD). Average nebulization process time per month, weighted by treatment scenario $(5 \%$ SABA alone, 75\% SABA/SAMA alone, 20\% SABA/SAMA + SABA PRN), was $32.1 \mathrm{~h}$ (Fig. 2b). A once-daily nebulized drug (80\% alone and $20 \%$ with once-daily SABA PRN) would require $13.7 \mathrm{~h}$ of nebulization process time per month, a $57 \%$ reduction (Fig. 2 b).

\section{Opportunity Cost (HCP Time Plus Materials)}

In both settings, nebulization materials (one tubing set and one mask or mouthpiece) are stored after nebulization and typically replaced after every nine uses. Applying national average salary data to time spent, average cost per nebulization for HCP time was $\$ 8.33$ (RT, inpatient setting) and $\$ 13.82$ (RN, LTC setting). Cost modeling of HCP time and materials in the inpatient setting yielded $\$ 243 /$ admission with current dosing versus $\$ 72 /$ admission with oncedaily dosing (70\% reduction). In the LTC setting, process cost was $\$ 1177 /$ month with current dosing versus $\$ 504 /$ month with once-daily dosing (57\% reduction).

\section{DISCUSSION}

The specific objectives of this research were to (1) document the healthcare professional's time 

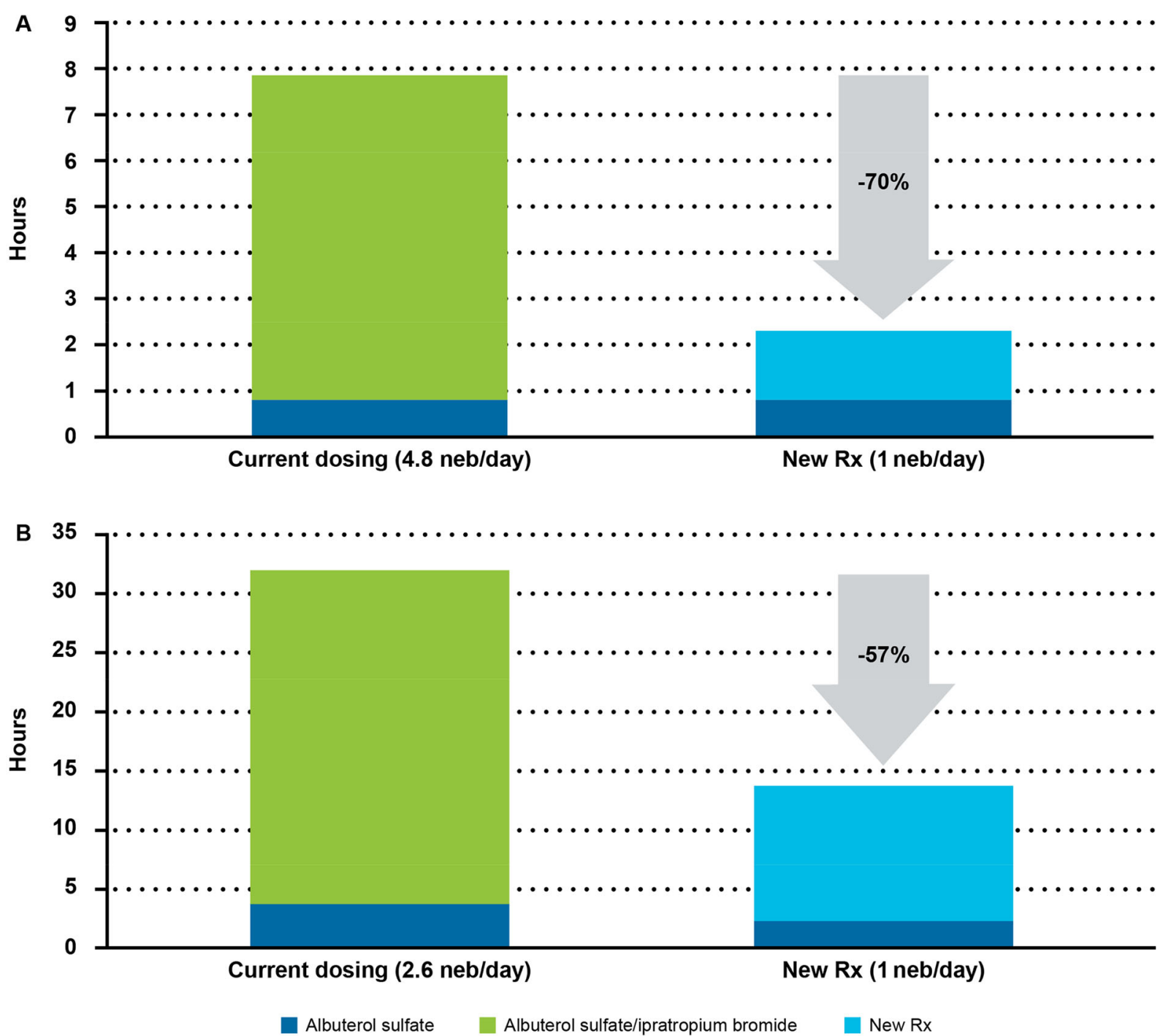

Fig. 2 a Nebulization process time per admission (inpatient). Weighted using reported distribution by regimen (40\% albuterol sulfate/ipratropium bromide alone, $60 \%$ albuterol sulfate/ipratropium bromide + albuterol sulfate PRN). Assumes 5.4 days per admission. b Nebulization process time per month (LTC). Weighted using reported

spent on all steps involved in the nebulization process of commonly used short-acting bronchodilators and (2) quantify (by modeling) potential efficiencies in labor time, and labor and material costs, from using less frequent dosing. The opportunity cost of the healthcare professional's time spent on nebulization-related activities demonstrates time that could be potentially freed up to be allocated to other distribution by regimen (5\% albuterol sulfate alone, $75 \%$ albuterol sulfate/ipratropium bromide alone, $20 \%$ albuterol sulfate/ipratropium bromide + albuterol sulfate PRN). LTC long-term care; neb nebulizer, $P R N$ as needed, $R x$ prescription

valuable COPD patient care activities. Given the focus of many providers solely on direct drug acquisition costs, such opportunity costs of time saved are often overlooked and not well studied, though they may significantly offset higher drug acquisition costs.

The choice of SABA/SAMA with or without SABA PRN as the standard of care treatment option was based on prior workflow mapping 
and semistructured interviews with clinical experts (site investigators and clinicians who oversee nebulized COPD therapy in their institutions), which indicated that SABA/SAMA with or without SABA PRN was the most commonly given nebulized COPD treatment for those patients benefiting most from nebulized therapy (e.g., patients with suboptimal peak inspiratory flow, patients with cognitive impairment) in the settings being studied (inpatient and LTC). The comparator used in the model was once-daily LAMA with or without SABA PRN; the model was not intended to be comprehensive of all potential alternative treatment scenarios (e.g., twice-daily LABA).

The nebulization process for COPD patients consumes substantial HCP time and associated costs over the course of an inpatient hospital admission or per month in the LTC setting. Findings from this pilot study validate the use of the T\&M methodology and suggest that switching from a SABA or SABA/SAMA combination to a once-daily nebulized drug could generate a sizable reduction in process time per admission or month of stay, depending on the setting and site characteristics.

Data were collected prior to the start of the COVID-19 pandemic, and thus the estimated time savings are likely conservative for the current environment. Since the start of the pandemic, additional steps have likely been added to the nebulization process, such as the use of personal protective equipment, specialized rooms, and new safety protocols. Accordingly, mean times for nebulization are likely greater than those reported in this study, and commensurately greater time efficiencies could be gained from nebulized therapies which have less frequent dosing. Furthermore, the COVID19 pandemic has caused additional staffing shortages and increased labor costs $[7,8]$, consequently magnifying the potential for cost savings with less frequently dosed nebulized treatments.

With a range of patient care tasks competing for RT and RN attention, there is a real opportunity cost associated with their time. Time released from nebulization-related activities can be allocated to other valuable care initiatives, such as patient education. With high demand for the time of RNs and RTs as a result of the pandemic, inpatient utilization of long-acting bronchodilators offers valuable time saved.

This pilot study has some limitations. The results reflect only a single site per setting and are therefore not generalizable across the country. Actual times for predefined tasks were measured, but in the model, the following variables were based on clinician opinion: distribution by COPD severity, expected nebulization frequency, and distribution by bronchodilator regimen. The cost modeling does not include the cost of the nebulized drugs, as this study was not intended to be a full cost analysis. A standard full cost analysis would incorporate consideration of drug acquisition cost and the costs of all other resources and outcomes involved in delivering nebulized COPD treatments. A wider analysis might also consider delivery of bronchodilators by handheld inhalers including metered dose inhaler and spacer. Such an analysis encompassing delivery mechanisms apart from nebulization could be an area of potential future study. The focus of our research specifically aimed to address the opportunity cost of HCP labor time spent on nebulization. The current analysis does not include any additional steps that may have been added to the nebulization workflow during the COVID-19 pandemic, such as donning and doffing of personal protective equipment for each nebulization, waiting periods around nebulization or designated nebulization rooms, and any other precautions that have been adopted.

This pilot study confirms the feasibility of T\&M methodology in this indication. It also provides support for conducting an expanded study involving additional study sites and a larger sample of observations that would increase the precision of the results, render them more generalizable to other inpatient and LTC settings across the US, and reflect any changes in workflow due to the pandemic.

\section{CONCLUSIONS}

In conclusion, the nebulization process for COPD patients in both inpatient and LTC 
settings consumes considerable HCP time and associated costs per inpatient hospital admission and per month of LTC stay. As patients with COPD often require extensive care and HCP time, interventions to improve COPD patient care efficiency without impacting quality are needed. A switch from SABA or SABA/ SAMA to a drug with a once-daily nebulization frequency could generate substantial time savings, depending on the setting of care and patient characteristics. Expanding this pilot study by adding additional sites and increasing the overall sample size would increase the precision of the results and make them more generalizable to inpatient and LTC settings across the US.

\section{ACKNOWLEDGEMENTS}

Funding. This study and the journal's Rapid Service Fee were funded by Theravance Biopharma Ireland Limited 269 (Dublin, Ireland).

Medical Writing, Editorial, and Other Assistance. Medical writing assistance was provided by Gautam Bijur, PhD, of Ashfield MedComms, an Ashfield Health company, and editorial assistance by Celia Nelson, also of Ashfield MedComms. Support for this assistance was funded by Mylan Inc., now merged with Upjohn to be Viatris, and Theravance Biopharma US, Inc.

Authorship. All named authors meet the International Committee of Medical Journal Editors (ICMJE) criteria for authorship for this article, take responsibility for the integrity of the work as a whole, and have given their approval for this version to be published.

Author Contributions. All authors listed on the title page contributed to the study concept and design; acquisition of data, analysis and interpretation of data; drafting of the manuscript; and critical revision of the manuscript for important intellectual content.
Disclosures. Brooks Kuhn is a speaker for Grifols and a consultant for Syneos Health. Grace Leung is a consultant for Theravance Biopharma US, Inc. Grant Maclaine was an employee of Theravance Biopharma Ireland Limited at the time of the study. Hemal Shah was a consultant for Mylan Specialty L.P. at the time of the study. Bryan Nichols is a contracted research pharmacist and has no affiliation or financial relationship to products or devices with a commercial interest related to the content of this publication. Erwin De Cock is an employee of Syneos Health commissioned by Theravance Biopharma US, Inc., to conduct this project.

Prior Presentation. This study was originally presented in part at the Eastern Pulmonary Conference, September 12-15, 2019, Palm Beach, FL.

Compliance with Ethics Guidelines. Institutional review board approval for 20 observations was obtained at each site; informed consent was not required because limited patient-level data (sex, COPD severity, cognitive status) were collected, and the focus of data collected was provider time. All data were anonymized. This study was performed in accordance with the Helsinki Declaration of 1964 and its later amendments.

Data Availability. All data generated or analyzed during this study are included in this published article.

Open Access. This article is licensed under a Creative Commons Attribution-NonCommercial 4.0 International License, which permits any non-commercial use, sharing, adaptation, distribution and reproduction in any medium or format, as long as you give appropriate credit to the original author(s) and the source, provide a link to the Creative Commons licence, and indicate if changes were made. The images or other third party material in this article are included in the article's Creative Commons licence, unless indicated otherwise in a credit line to the material. If material is not included in the article's Creative 
Commons licence and your intended use is not permitted by statutory regulation or exceeds the permitted use, you will need to obtain permission directly from the copyright holder. To view a copy of this licence, visit http:// creativecommons.org/licenses/by-nc/4.0/.

\section{REFERENCES}

1. American Lung Association. Understanding COPD Trends: morbidity and mortality. https://www.lung. org/blog/understanding-copd-trends. Accessed June 7, 2021.

2. Duarte AG, Tung L, Zhang W, et al. Spirometry measurement of peak inspiratory flow identifies suboptimal use of dry powder inhalers in ambulatory patients with COPD. Chronic Obstr Pulm Dis. 2019;6(3):246-55. https://doi.org/10.15326/jcopdf.6. 3.2018.0163.

3. US Bureau of Labor Statistics. Occupational outlook handbook: respiratory therapists. https://www.bls. gov/ooh/healthcare/respiratory-therapists.htm. Accessed June 7, 2021.

4. De Cock E, Leung G, Maclaine G, et al. Workflow mapping of nebulized COPD therapy in inpatient and long-term care (LTC) settings in the US: a precursor to an observational time and motion (T\&M) study. In: Poster presented at: Eastern Pulmonary Conference; September 12-15, 2019; Palm Beach, FL.

5. US Bureau of Labor Statistics. Occupational employment and wages, May 2020. https://www.bls.gov/oes/ current/oes291141.htm. Accessed June 7, 2021.

6. US Bureau of Labor Statistics. Occupational employment and wages, May 2020. https://www.bls.gov/oes/ current/oes291126.htm. Accessed June 7, 2021.

7. $\mathrm{Xu} \mathrm{H}$, Intrator O, Bowblis JR. Shortages of staff in nursing homes during the COVID-19 pandemic: what are the driving factors? J Am Med Dir Assoc. 2020;21(10):1371-7. jamda.2020.08.002. https://doi.org/10.1016/j.

8. Scales K. It is time to resolve the direct care workforce crisis in long-term care. Gerontologist. 2021;61(4): 497-504. https://doi.org/10.1093/geront/gnaa116. 